\title{
The effect of refractive error on central and peripheral motion sensitivity at various exposure durations
}

\author{
R. B. POST and H. W. LEIBOWITZ \\ Pennsylvania State University, University Park, Pennsylvania 16802
}

\begin{abstract}
The influence of refractive error on movement sensitivity was determined for a range of stimulus durations in the fovea and periphery. At short durations, threshold is determined by a constant displacement of the stimulus. At longer durations, a constant velocity is required. The correction of peripheral refractive error increases movement sensitivity in the displacement (short duration) component, but does not influence sensitivity in the velocity (long duration) component of the motion threshold. Implications concerning the mechanisms underlying motion perception are discussed.
\end{abstract}

The presence of refractive error, or blur, is known to influence most visual functions in the fovea. Response measures such as acuity or stereoscopic resolution are altered systematically by variations in the amount of refractive error present. Since acuity is so important in our society, a large proportion of visual health care is devoted to the correction of refractive error for foveal viewing.

Large and variable refractive errors are also commonly present in peripheral vision even when the foveal refraction has been corrected (Ferree \& Rand, 1933), although their significance for visual functioning has not been fully determined. Unlike foveal acuity, peripheral acuity improves only slightly or not at all with correction of peripheral refractive error (Frisen \& Glausholm, 1975; Millodot, Johnson, Lamont, \& Leibowitz, 1975). It is possible that the relatively high spatial frequency information lost due to refractive error has less effect on peripheral acuity because of the poor resolution of the peripheral retina. Thus, refractive error may remove only information to which the peripheral retina is insensitive.

In contrast with the findings for peripheral acuity, Johnson and Leibowitz (1974) and Leibowitz, Johnson, and Isabelle (1972) report that peripheral movement detection improves with correction refractive errors. Although this improvement was found consistently under a variety of conditions, the use of a stimulus duration of $1 \mathrm{sec}$ limits the generality of the findings. This limitation is apparent from other investigations that have demonstrated threshold movement to be

This research was supported by Grants MH08061 from the National Institute of Mental Health and EY03276 from the National Eye Institute. The authors express their appreciation to $\mathrm{L}$. Kerr and B. U. Crassini for a critical reading of the manuscript. R. B. Post's current address is: Department of Ophthalmology, University of California, Davis, California 95616. limited by different stimulus features at durations above and below about $1 \mathrm{sec}$. At durations of about $1 \mathrm{sec}$ or less, threshold movement has been found to depend on a constant minimal displacement of the stimulus (Dimmick \& Karl, 1930; Henderson, 1971; Leibowitz, 1955). At longer durations, however, a constant minimal velocity is required (Johnson \& Leibowitz, 1976). Since it is likely that motion detection is limited by different factors above and below $1 \mathrm{sec}$, one may not generalize the findings obtained with $1-\mathrm{sec}$ exposures to other durations. ${ }^{1}$ It is possible that the observed influence of refractive error in limiting motion sensitivity occurs only for a mechanism that operates at either short or long durations, but not at both.

The purpose of the present experiment was to determine the relative influence of naturally occurring refractive error on the mechanisms mediating motion detection at both short and long durations. It would be expected that the blurred stimulus contour resulting from uncorrected refractive error would have the greatest consequence when the resolution of small spatial extents is involved, that is, at short durations in which a small displacement of the stimulus determines motion perception. The shallow luminance gradient at the edge of a blurred stimulus would have to travel farther than a steep luminance gradient in order to produce similar responses. The presence of blur would therefore be expected to increase the smallest detectable stimulus excursion. At longer durations, however, the distortions of stimulus contour caused by refractive error are small relative to the large stimulus excursions involved. At these durations, the operation of mechanisms sensitive to changes in the overall position of the stimulus might be expected to be relatively independent of the optical quality of the stimulus contour, since blur does not affect the apparent location of the stimulus in the field (Post \& Leibowitz, 1980). 


\section{METHOD}

\section{Subjects}

All four subjects (aged 19-27) had prior experience performing psychophysical tasks and exhibited a minimum of 20/20 (corrected) near and far visual acuity in the right (viewing) eye as determined by $\mathbf{a}$ Bausch and Lomb orthorater.

\section{Apparatus}

Subjects were seated while they viewed monocularly the interior of a perimeter $77 \mathrm{~cm}$ in radius. The interior of the perimeter was covered with black flock paper with a reflectance of $.85 \%$ and illuminated by a panel of incandescent lights (luminance $=$ $.14 \mathrm{~cd} / \mathrm{m}^{2}$ ). A slit, $1.27 \mathrm{~cm}$ in height, extended horizontally the width of the perimeter at the subject's eye level. An adjustable chinrest maintained a stable head position. Fixation was maintained by means of a blind-spot fixation technique (Teuber, Battersby, \& Bender, 1960). A strip of flat white tape, $1.25 \times 5.1 \mathrm{~cm}$ (reflectance $=65.5 \%$, luminance $=3.42 \mathrm{~cd} / \mathrm{m}^{2}$ ) and subtending an angle of $.93 \times 3.80 \mathrm{deg}$, was attached to the interior of the perimeter, with the long axis aligned horizontally and centered $15 \mathrm{deg}$ from the center of the perimeter. With stable fixation on the center of the perimeter, this strip, located in the blind spot of the right hemifield, was not visible and appeared only when fixation shifted. Trials during which the strip became apparent to the subject were discarded. A lens holder was suspended from the ceiling of the perimeter, which allowed placement of ophthalmic trial lenses. The orientation of the holder was adjustable for different viewing eccentricities.

The motion stimulus was generated by wrapping a strip of flat white tape $\left(\right.$ reflectance $=65.5 \%$, luminance $\left.=3.42 \mathrm{~cd} / \mathrm{m}^{2}\right)$, $1.27 \mathrm{~cm}$ wide, in a helix around a black drum (reflectance $=.85 \%$ ), which was mounted on the external wall of the perimeter. Rotations of the drum about its axis produced horizontal displacements of an approximately square-shaped portion of tape (subtending $.95 \mathrm{deg}$ of visual angle) visible through the perimeter slit. The axis of the drum was attached to a synchronous de motor and 10-ratio gear reducer that permitted variations in the speed and direction of drum rotation. Duration of rotation was regulated by a Hunter timer. The location of the drum was adjustable, producing different eccentricities of the target.

\section{Procedure}

Since determinations of peripheral motion thresholds typically show practice effects (Johnson \& Leibowitz, 1974), the first phase of this experiment provided the subjects with experience in making motion judgments. Four practice sessions were given at each of three target eccentricities: 0,30 , and $60 \mathrm{deg}$. At the end of these sessions, stable performance had been achieved at each eccentricity. During each session, velocity thresholds were determined for stimulus durations of $.1, .25, .5,1.0,2.5$, and $5.0 \mathrm{sec}$, by means of a staircase procedure using blocked trials. Each block contained 10 trials of one stimulus velocity. The velocity of each block varied in a staircase manner with variable step size until velocities were found that bracketed a $75 \%$ correct level of responding. The velocity corresponding to a $75 \%$ correct level of response was then interpolated and used as the threshold value. Prior to each trial, the experimenter said "ready" to assist the subject in fixating. On each trial, the stimulus moved to either the left or the right, and the subject indicated the perceived direction of movement. About 40 to 50 trials were typically required for each velocity threshold determination.

Near the end of this series of practice sessions, the spherical and astigmatic errors present at each viewing eccentricity were determined for the four subjects. Refractions were performed in the apparatus by an experienced optometrist by means of streak retinoscopy. The corrections determined for each subject at each eccentricity appear in Table 1.

The remainder of the experiment consisted of six experimental
Table 1

Optical Corrections, in Diopters, Required by Individual Subjects at Each Stimulus Eccentricity

\begin{tabular}{ccccc}
$\begin{array}{c}\text { Sub- } \\
\text { ject }\end{array}$ & $\begin{array}{c}\text { Eccen- } \\
\text { tricity }\end{array}$ & $\begin{array}{c}\text { Spher- } \\
\text { ical }\end{array}$ & $\begin{array}{l}\text { Cylin- } \\
\text { drical }\end{array}$ & $\begin{array}{c}\text { Cylinder } \\
\text { Axis }\end{array}$ \\
\hline & 0 & +1.00 & -.50 & 90 \\
G.H. & 30 & +2.25 & -1.25 & 90 \\
& 60 & +5.50 & -3.00 & 90 \\
& 0 & 0.00 & -.50 & 105 \\
T.B. & 30 & +2.00 & -1.00 & 105 \\
& 60 & +6.00 & -1.75 & 110 \\
& 0 & -1.25 & 0.00 & \\
S.W. & 30 & +1.75 & -1.00 & 105 \\
& 60 & +6.50 & -1.50 & 110 \\
& 0 & +1.00 & -1.00 & 180 \\
R.P & 30 & +1.00 & -1.00 & 180 \\
& 60 & +1.25 & -1.25 & 180 \\
\hline
\end{tabular}

sessions, during which motion thresholds were determined in the same manner as during practice with and without correction of refractive errors. During each session, thresholds for three stimulus durations were determined at one eccentricity, both with and without refractive error. The order of corrected, as opposed to noncorrected, determinations was counterbalanced. During the refractive error-corrected conditions, the lens holder with the appropriate spherical and astigmatic lenses was adjusted to the stimulus eccentricity. For the uncorrected conditions, a pair of plano lenses replaced the corrections.

\section{RESULTS}

The mean values of the movement thresholds for all subjects at each eccentricity are plotted in Figure 1, which presents threshold displacements on a double logarithmic plot as a function of duration. The displacements were determined by multiplying the threshold velocity measures by the stimulus duration. This format facilitates comparison of the obtained values with those expected from the operation of different limiting factors. A horizontal line indicates that threshold is determined by a constant minimal displacement; that is, motion was accurately reported only when the stimulus had moved a critical distance independent of velocity. The dependence upon a constant velocity, however, results in a line of unit slope, because displacement increases directly with duration when velocity is held constant. The theoretical functions expected from these limits are plotted in Figure 1. (The inflection is located at $1.0 \mathrm{sec}$ to correspond to the results of previous experiments.) As expected, under all experimental conditions the data from durations of less than $1 \mathrm{sec}$ approximate the horizontal line representing constant displacement, while the values obtained at longer durations more closely approximate the constant velocity prediction.

A comparison of the values obtained at different viewing eccentricities reveals that sensitivity decreases with increasing eccentricity at all stimulus durations. For the fovea, threshold is determined by a displace- 

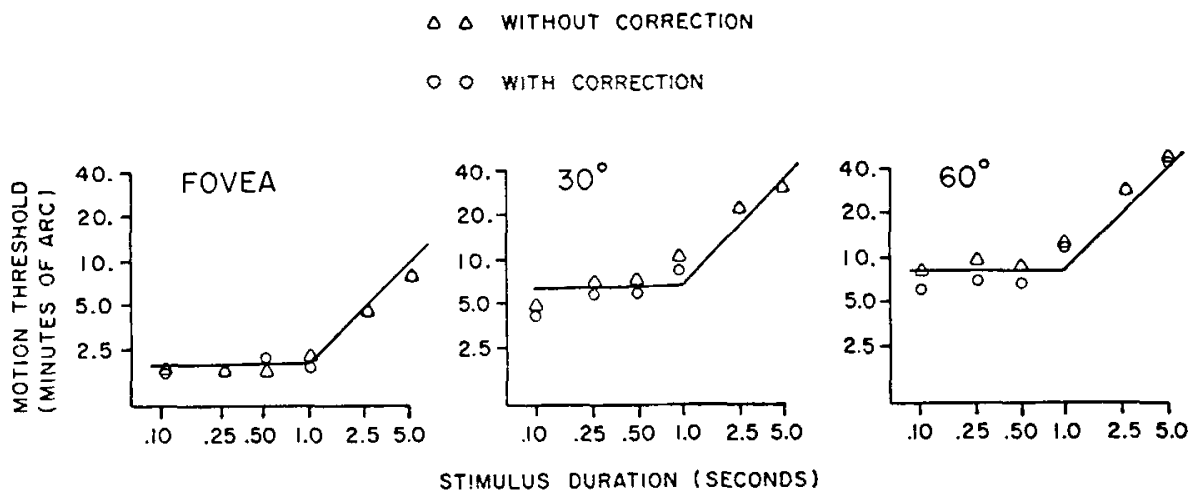

Figure 1. Threshold displacements obtained with and without correction of refractive error at three eccentricities.

ment of the stimulus through $1.75 \mathrm{~min}$ of arc for durations up to $1 \mathrm{sec}$. At longer durations, a velocity of about $1.6 \mathrm{~min}$ of arc/second is required. These values are systematically elevated in the periphery. At 60 degs eccentricity, the displacement required at durations under $1 \mathrm{sec}$ is about $6.7 \mathrm{~min}$ of arc for the refractive error-corrected condition and $8.7 \mathrm{~min}$ for the uncorrected case. Beyond $1 \mathrm{sec}$, a velocity of $9.5 \mathrm{~min} / \mathrm{sec}$ is required for both corrected and uncorrected viewing conditions.

The influence of refractive error varies with both stimulus eccentricity and duration. Foveal sensitivity is nearly identical under conditions of corrected and uncorrected refractive error. Sensitivity at long durations in the periphery is likewise shown to be independent of refractive state. At short durations in the periphery, however, the correction of refractive error facilitates motion detection. The horizontal portions of the peripheral thresholds are lowered slightly across durations of .1 to $1.0 \mathrm{sec}$, indicating a decrease in the extent of the constant displacement required for motion detection at short durations.

At each eccentricity, the effects of refractive error were analyzed separately for short $(.1$ to $1.0 \mathrm{sec})$ and long $(2.5$ and $5.0 \mathrm{sec})$ durations. Analysis of the difference between corrected and uncorrected threshold values for each subject by means of a Wilcoxon signedrank procedure reveals that refractive error significantly influences motion sensitivity for short durations at both $30 \operatorname{deg}(Z=2.98, p<.01)$ and $60 \operatorname{deg}(Z=3.28$, $p<.001)$. No effects of refractive error are found in the fovea or at long durations in the periphery. The results of this analysis confirm the pattern that is apparent in Figure 1.

\section{DISCUSSION}

The present findings demonstrate the limiting effects of different factors upon motion sensitivity. At durations of less than $1 \mathrm{sec}$, a constant displacement of the stimulus is required for the detection of movement.
This displacement limit has previously been well documented (Cohen \& Bonnet, 1972; Dimmick \& Karl, 1930; Henderson, 1971; Johnson \& Leibowitz, 1976; Leibowitz, 1955). At longer durations, however, a constant velocity is required, in agreement with the observations of Harvey and Michon (1974) and Johnson and Leibowitz (1976). The present finding that similar effects of duration occur in the fovea and the periphery has also been previously reported by Johnson and Leibowitz (1976). The correction of naturally occurring refractive errors is found to improve peripheral motion detection at short durations, since the displacement required for detection decreases with the correction of optical blur. However, no effects of blur were found with foveal viewing at any duration or at long durations in the periphery.

The findings that refractive errors influence motion sensitivity at short durations would suggest that the mechanisms mediating detection at these durations are responding to some stimulus feature that is altered by refractive error: One such feature is the slope of the luminance gradient forming the contour, which is degraded when blurred. Therefore, a mechanism that responds to a given displacement of a sharp edge might be expected to require a greater displacement of a degraded edge in order to elicit a threshold response, since the degraded edge would have to travel farther before it would produce an equivalent change in luminance at a given retinal location.

The lack of an effect of refractive error at longer durations, however, suggests that the mechanisms mediating motion detection at these durations are responding to some stimulus feature that does not change with blur. One such feature is the location or position of the center of the stimulus. A given movement of a stimulus results in precisely the same change in location of the center of the stimulus, regardless of the quality of the contour defining the edges of the stimulus. The different results obtained at short and long durations would, therefore, indicate the operation of different mechanisms at these durations. 
Johnson and Leibowitz (1974) and Leibowitz, Johnson, and Isabelle (1972) had previously observed the limiting effect of dioptrics upon motion detection at stimulus dúrations of $1 \mathrm{sec}$. It should be noted that the improvement obtained in the present study is smaller than that previously reported. While we are not certain of the basis for this difference, it is perhaps attributable to the use of a two-alternative forcedchoice procedure in the present study. Also, comparison of the values obtained in the three studies indicates that subjects in the present study displayed greater uncorrected sensitivity. Additionally, in none of the studies were foveal motion thresholds found to be sensitive to refractive error. It is possible that, during the experiments, the young subjects were able to compensate for slight refractive errors by accommodating to the motion stimulus that was not present during the refraction procedure.

The results obtained at short durations in this experiment demonstrated the limiting effect of dioptrics upon motion detection in the periphery. Dioptrics have likewise been shown to influence peripheral increment detection (Fankhauser \& Enoch, 1962) and determinations of the Westheimer function (Enoch, Sunga, \& Bachman, 1970; Sunga \& Enoch, 1970). Peripheral acuity, on the other hand, has been shown to be independent of, or only slightly affected by, spherical and astigmatic errors (Frisen \& Glausholm, 1975; Millodot, Johnson, Lamont, \& Leibowitz, 1975). Comparison of the acuity findings with the results obtained for motion sensitivity at short durations is interesting, as it has been proposed (Gordon, 1947) that similar mechanisms serve to limit both acuity and the resolution of threshold movements. The finding that blur disrupts peripheral motion detection at short durations but not peripheral acuity suggests that different stimulus features and mechanisms subserve these two functions.

Based on this and previous studies, it can be concluded that refractive error plays only a limited role in limiting peripheral sensitivity. Only for durations of $1 \mathrm{sec}$ or less in which small displacements limit motion sensitivity are refractive errors found to influence the movement threshold. At longer durations, however, the motion threshold, like acuity, is uninfluenced by the correction of naturally occurring refractive errors.

\section{REFERENCES}

Cohen, R., \& Bonnft, C. Movement detection thresholds and stimulus duration. Perception \& Psychophysics, 1972, 12, 269-272.

Diммıск, F. L., \& KaнL, J. C. The effect of the exposure time upon the R. L. of visible motion. Journal of Experimental Psychology, 1930, 13, 365-369.

Enoch, J., Sunga, R., \& Bachman, E. A static perimetric technique believed to test receptive field properties: 1. Extension of the Westheimer experiments on spatial interaction. American Journal of Ophthalmology, 1970, 69, 113-126.

Fankhauser, F., \& ENOCH, J. The effects of blur on perimetric thresholds. A.M.A. Archives of Ophthalmology, 1962, 68, 240-251.

Ferree, C. W., \& RAND, G. Interpretation of refractive conditions in the peripheral field of vision. A.M.A. Archives of Ophthalmology, 1933, 9, 925-938.

Frisen, L., \& Glausholm, A. Optical and neural resolution in peripheral vision. Investigative Ophthalmology, 1975, 14, 528-536.

Gordon. D. The relation between the thresholds of form, motion and displacement in parafoveal and peripheral vision at a scotopic level of illumination. American Journal of Psychology, 1947, $60,202-225$

Harvey, L., \& Michon, J. Detectability of relative motion as a function of exposure duration, angular separation and background. Journal of Experimental Psychology, 1974, 103, 317-325.

Henderson, D. The relationships among time, distance, and intensity as determinants of motion discrimination. Perception \& Psychophysics, 1971, 10, 313-320.

Johnson, C., \& Leıbowitz, H. Practice, refractive error, and feedback as factors influencing peripheral motion thresholds. Perception \& Psychophysics, 1974, 15, 276-280.

Johnson, C., \& Leibowitz, H. Velocity-time reciprocity in the perception of motion: Foveal and peripheral determinants. Vision Research, 1976, 16, 177-180.

LEiBowitz, $H$. The relation between the rate threshold for the perception of movement and the luminance for various durations of exposure. Journal of Experimental Psychology, 1955, 49, 209-214

Leibowitz, H., Johnson, C., \& Isabelle, E. Peripheral motion detection and refracive error. Science, 1972, 177, 1207-1208.

Millodot, M., Johnson, C., Lamont, A., \& Leibowitz, H. Effect of dioptrics on peripheral visual acuity. Vision Research, $1975,15,1357-1362$.

Post, R., \& Leibowitz, H. The independence of radial localization from refractive error. Journal of the Optical Society of America, $1980,70,1377-1379$.

Sunga, R., \& ENoch, J. Further perimetric analysis of patients with lesions of the visual pathways. American Joumal of Ophthalmology, 1970, 70, 403-422.

Teuber, H., Battersby, W., \& Bender, M. Visual field defects after penetrating missile wounds of the brain. Cambridge, Mass: Harvard University Press, 1960.

\section{NOTE}

1. The possibility that different mechanisms are operable for long and short durations is further supported by recent observations on residual motion sensitivity in a cortical scotoma (Riddoch phenomenon). Although motion sensitivity in the scotomatous field is degraded at all durations of exposure, the impairment is substantially greater for brief (under one second) as compared with long duration stimuli. (Psychophysical analysis of motion sensitivity in the Riddoch phenomenon, R. B. Post and H. W. Leibowitz, in preparation).

(Received for publication July 2, 1980; revision accepted November $18,1980$. 